\title{
The Reality of Making a Medication Administration Error in Nursing Practice: Nurses Share Their Lived Experiences
}

\section{Seema Lall}

Harriet Rothkopf Heilbrunn School of Nursing, Long Island University, Brooklyn, New York, USA

\begin{abstract}
The complexity of clinical nursing practice, chaotic and technical nature of the workplace environment coupled with the multiple and varied roles of nurses, leads to cognitive overload that may overwhelm nurses, which may result in medication errors. All medication errors are considered serious events, but some may consequently be harmful to patients and have a lasting effect on the nurses involved in making the error. This study examined what it was like to make a medication error for registered nurses. A descriptive phenomenological study rooted in the philosophical tradition of Husserl [1] was conducted on eight registered nurses via two in-depth face to face interviews. The data generated from a total of sixteen interviews and field notes were analyzed using Colaizzi's seven-step method. Five theme categories emerged: Immediate Impact: Psychological and Physical Reactions; Multiple Causes within Chaos: Cognitive Dimensions; Embedded Challenges: Healthcare Setting; Organizational Culture: Within the Place/Within the Person; Dynamics of Reflection: Looking Forward. The lived experience of making a medication administration error led nurses to the realization that a profound occurrence had taken place. As a result, these nurses experienced upheavals that were of a physical and emotional nature, which threatened their professional status and generated a sense of low self-esteem that considerably decreased their selfconfidence. An overwhelming amount of workload, a stressful work environment and mistreatment by peers were predominantly noted as the factors that led to these errors. Nurses in this study offered suggestions to improve the system but felt their concerns were often undervalued. Implications for nursing education and nursing practice, to significantly improve teaching strategies of medication administration process leading to improved patient outcomes, were suggested.
\end{abstract}

Publication History:

\section{Background}

One of the key roles that nurses perform while delivering quality care to patients is safe and accurate medication administration. Nurses are well versed with the concept of safe patient care and understand that responsibility and accountability of administering medication to patient/s ultimately rests with the person (nurse) who administers the medication. The importance of accurate medication administration is emphasized in the nursing profession and well understood by practicing nurses. It is therefore, understandable that nurses are apprehensive when a medication error/s occurs, specifically, because the error may be viewed by others as a violation of the ethical aspect of non-maleficence with respect to patient welfare and poses a threat to the nurses involved in terms of blame, potential loss of position/ job and much more. Nonetheless, one must appreciate that despite the medication error occurrence, nurses continue to care for patients as well as deal with their individual feelings of being involved in the medication error incident each day. Research suggests that medication error incidents particularly prove to be extremely stressful for these nurses and may have a devastating effect on them which may be long lasting and may be considered 'second victims' of medication error occurrences $[2,3]$.

\section{Significance}

Patient safety continues to be a matter of concern for nurses, nurse leaders and administrators. This was first substantiated by the Institute of Medicine (IOM) through their first report released in the year 2000, entitled 'To Err Is Human: Building a Safer Healthcare System'. Subsequently, over time IOM published multiple reports that underscored that medication errors were the leading and most common predictable cause for adverse events in healthcare facilities, and that administration errors were placed as the most common identifiable medical errors in the USA. This additionally, called attention of healthcare professionals, nurse leaders and nurses to the high rates of negative patient outcomes, some of which were quite harmful to the patients and may have costed them their lives [48]. Literature reviewed over two decades revealed that medication errors occur frequently in hospitals and may prove to be fatal and costly. A study published in the United States on medical errors, highlighted that out of 48,000-98,000 deaths that were related to drug complications, about 7000 deaths were related to medication errors annually [9]. Another study reported that administration errors account for approximately $59 \%$ of all medication errors and one out of every three adverse drug events (ADEs) is related to medication errors. A study conducted in two teaching hospitals revealed that about $2 \%$ of patients admitted experienced preventable ADEs, costing the hospital approximately $\$ 4,700$ per admission or nearly $\$ 2.8$ million annually for a 700-bedded teaching hospital and if these findings could be generalized then the preventable ADEs are costing the country approximately $\$ 2$ billion per year. A 1997 study reviewed admissions in a large teaching hospital that indicated 2.43 of the admissions out of 100 admissions were complicated due to an ADE. This led to an increase in the length of stay (LOS) of these patients by 1.91 days escalating the cost to the hospital by $\$ 2,262$. Additionally, ADEs indirectly impact costs for loss of productivity, disability and other care related costs $[4,10-13]$.

"Corresponding Author: Dr. Seema Lall, Harriet Rothkopf Heilbrunn School of Nursing Long Island University (Brooklyn campus), 373, 92 street Apt A 58, Brooklyn, New York, 11209, USA, Tel: 347-393-9240; E-mail: lall_seema@yahoo.com

Citation: Lall S (2018) The Reality of Making a Medication Administration Error in Nursing Practice: Nurses Share Their Lived Experiences. Int J Nurs Clin Pract 5: 286. doi: https://doi.org/10.15344/2394-4978/2018/286

Copyright: (c) 2018 Lall. This is an open-access article distributed under the terms of the Creative Commons Attribution License, which permits unrestricted use, distribution, and reproduction in any medium, provided the original author and source are credited. 


\section{The Study}

The purpose of this study was to examine and understand the lived experience of nurses' involvement in making one or more medication errors that reached the patient, may have resulted in negative patient outcomes. This study also attempted to gain a unique understanding of how these nurses coped with the aftermath, as well as to more and fully understand the support nurses may need after the occurrence.

Research questions:

'What is the lived experience of nurses involved in medication errors?'

'What is the lived experience of these nurses caring for patients after the medication error occurred?'

'What is the lived experience of these nurses working on the unit after the medication error occurred?'

\section{Design and methodology}

A qualitative descriptive phenomenology rooted in the philosophical tradition of Husserl was conducted. Two interviews were carried out with each participant and the research data were generated from a total of sixteen interviews and field notes. Colaizzi's seven-step methodological guidelines were used for analysis and interpretation of data.

\section{Participant recruitment and profile}

Participants for this study were registered nurses (RNs) employed in facilities and experienced in caring for patients in a variety of healthcare areas. These nurses were currently practicing with a valid RN license for at least six months in the state of New York. A purposive sample of eight RNs with the above inclusion criteria who were directly involved in one or more medication errors were selected. The recruitment of participants was primarily done by snowballing method. The first participant however, was recruited by posting a flyer on the announcement board of a RN- BS completion program. The study sample comprised of seven female and one male participant with ages ranging from 36-55 years. The educational background of one participant was associate degree in nursing; five held a baccalaureate degree and two were masters prepared RNs. Their professional experience of practicing as RNs ranged from 3-22 years. Two participants prior to practicing as RNs also had the opportunity to practice as licensed practical nurses (LPNs). Most of the participants had the opportunity to practice in more than one area of expertise. The work areas of these participants were: medicalsurgical, neurology intensive care unit, emergency department, telemetry, intensive care unit, nursing home and home care. Most of the participants were full time registered nurses in one facility with a second part-time job in another facility. Two participants worked as per diem RNs in their second job and floated to a different unit almost every day. Of the eight participants, three participants worked eighthour day shifts, one worked twelve-hour day shift and one worked both day and night eight-hour shifts. The remaining three participants worked twelve-hour night shifts (table 1 below shows participant demographics).

\section{Data collection}

Data collection was done by the principle investigator via two faceto-face interviews. The first interview was an in-depth interview with open-ended questions; the second being a focused interview with probes to clarify information obtained during the first interview. This style of interviewing allowed the researcher to follow the participant's lead to format clarifying questions for the second interview [14]. The researcher carefully observed participants' during the interviewing process for any nonverbal expressions and the elements of their environment. The observations were recorded in the field notes. The researcher encouraged each participant to describe his or her lived experience without leading the discussion. Broad open-ended questions were used in the first interview to allow participants to gradually ease into the interviewing process.

Examples of questions included in the first interview were: 'Describe the incident when you were involved in a medication error'. 'Describe your feelings when you made the medication error'. Probes were used in the second interview, examples of these were: 'What was it like to care for the patient involved in the incident?' 'What were the reactions of your colleagues/supervisor after the incident?' Based on the literature review the interview questions were structured to focus on specific areas like:

- Nurses' reactions (physical, emotional and psychological) to medication error occurrence immediately after the incident, and later.

- Factors within practice environment that led to medication error occurrences.

- Challenges faced by nurses before during and after medication error occurrence.

\begin{tabular}{|l|l|l|l|l|l|l|}
\hline Participant & Gender & Age in years & Years of experience & Education & $\begin{array}{l}\text { Unit worked on } \\
\text { Nurse: patient ratio }\end{array}$ & $\begin{array}{l}\text { Shift/Time of day } \\
\text { Length/Status }\end{array}$ \\
\hline P1 & Female & 43 & 10 & BSN & Medical-Surgical (NH) / 1:40 & Day/8 hr/FT, Float \\
\hline P2 & Female & 39 & 3 & BSN & Neuro ICU/ 1:2 & Night/12 hr/FT \\
\hline P3 & Female & 39 & 3 & BSN & ER/1:12 or 3;24 & Night $/ 12 \mathrm{hr} / \mathrm{FT}$ \\
\hline P4 & Female & 47 & 12 & BSN, MS & Medical-Surgical (NH) $/ 1: 38$ & Day $/ 8 \mathrm{hr} / \mathrm{Per} \mathrm{Diem}$ \\
\hline P5 & Female & 55 & 22 & BSN, MPH & Medical-Surgical (NH) $/ 1: 20$ & Day $/ \mathrm{Night} / 8 \mathrm{hr} \mathrm{FT}$ \\
\hline P6 & Female & 36 & 9 & BSN & Telemetry $/ 1: 8$ (home care) & Day $/ 12 \mathrm{hr} / \mathrm{FT}$ \\
\hline P7 & Female & 43 & 12 & BSN & Medical-Surgical $/ 1: 10$ & Night $/ 12 \mathrm{hr} / \mathrm{FT}$ \\
\hline P8 & Male & 51 & 10 & ADN & Telemetry $/ 1: 6$ & Day/8 hr/FT \\
\hline
\end{tabular}

Table 1: Participant Description by Demographics. 
- Culture within the work area and /or factors that predisposed to medication error occurrences.

- Coping mechanisms used by nurses when medication error/s occurred.

- Type of support needed by nurses after the medication error occurrence.

- Nurses' perceptions as to why the medication error occurred.

- Nurses' reflections about error occurrence and effects on future practice.

Each interview lasted approximately 50-60 minutes. These were conducted in a private area of choice of each participant. The duration of data collection process was about three months. Being that all except the first participant were recruited by snowballing method, the interviews were conducted as participants were recruited. The second interview was scheduled by setting a date approximately one week later immediately after the first interview was completed. The date was scheduled according to the convenience of the participants. All interviews were conducted by the principal investigator and completed as scheduled except participant \#3, whose second interview was scheduled after three weeks due to an illness. Even though questions for the two interviews were previously formatted to gain basic information, the researcher (interviewer) reformatted interview questions to make their meanings explicit to respondents as needed.

\section{Trustworthiness and rigor}

Techniques to ensure trustworthiness were employed by engaging participants, member checking, thick descriptions, and audit trail methods for this study. Participant engagement was accomplished by building a relation of trust between the investigator and the participant. The researcher gave relevant explanations and answered any questions the participants had prior to interview, enabling comfort and gradual ease into the interview process. Member checking involved the action of returning of the investigator to each participant after the interview process was complete to ensure correctness of the information given by the participant and to validate the essential structure developed for the preservation of the true meaning of the experiences [15]. The researcher made the participant aware of this at the time of interview sessions. All participants responded via email. Thick descriptions were key to the meaning of research and an important aspect of trustworthiness. The researcher sought permission and verification to use narratives, which was crucial for this study. Finally, audit trail was critical to establishing authenticity and trustworthiness of the data. The researcher documented the research activities over time with clarity and in a systematic way to be followed by others to understand the line of thinking used by researcher at the time of data analysis $[16,17]$. To ensure methodological rigor the researcher used the processes of phenomenological reduction, bracketing and member checking. The researcher's attitude was non-judgmental, she practiced the art of openness as the data were revealed, which was carried out before the study began and continued throughout data collection and data analysis [14].

\section{Ethical considerations}

The study was approved by Institutional Review Board, Adelphi University, New York. The researcher obtained a written informed consent from each participant prior to data collection. The participants were assured of confidentiality and the right to withdraw from the study at any time during the research. Anonymity was not completely assured, as the researcher was the interviewer however, the participants' names were coded to allow for confidentiality when data was examined by experts. Since the study investigated error in the participants' workplaces, all methods to eliminate and/or avoid coercion were employed always during the research. Permission to tape record the interviews was obtained prior to each interview being conducted. To ensure participant privacy and comfort the interviews were conducted in a private area that allowed for a calm environment [14]. A "cooling-off" period was provided to individual participants before moving the interview forward in case of any emotional upset or distress during the interview process while they reflected upon their experiences of medication errors. Individual participants were also provided the opportunity to reschedule the interview if needed.

\section{Data analysis}

The recorded interviews were transcribed verbatim by the researcher. Transcription of interviews was completed in approximately two months. The data collected from the eight participant's interviews revealed ten medication errors which were categorized as: unauthorized drug error, wrong route error, wrong dose error, omission error and wrong documentation error (table 2). The interviewer also gathered additional data in the form of field notes for all eight participants. Data were subjected to intense and thorough analysis utilizing Colaizzi's method, significant statements were extracted and in-depth explanation of the meaning of each statement was achieved after coding the data. This process was valuable in understanding the actual meaning behind each statement made by the participants. Coding and intense analysis of data resulted in two hundred and thirty formulated meanings. All data were coded by hand by the researcher. As the process of coding and recoding continued previously examined codes were re-examined for new codes and repetitions retained for each protocol. The transcripts were reviewed with a doctorally prepared nurse researcher for authenticity and validation of the coding system. Significant statements were subjected to systematic review, formulated meanings were carefully constructed to preserve the context of the protocol as closely as possible to the stated expressions to ensure preservation and significance of their meaning. Formulated meanings were organized into clusters of themes and theme categories. Themes were continuously referred back to the original protocols to ensure validation and commonality of themes.

The data were finally organized into nineteen theme clusters and five theme categories. The findings presented are the nurses' (participants) descriptions of the reality of their lived experiences of making one or more medication errors. The actuality of the meaning was derived from the experiences that these nurses lived through during and after the medication error ocurred. Following are the descriptions derived from the lived experiences of eight registered nurses and are presented under five theme categories.

\section{Theme category 1- Immediate Impact: Psychological and Physical Reactions}

Nurses described the immediate reactions of mind and body experienced by them when a medication error was committed. They verbalized feelings of fear, anxiety, remorse, disappointment, unbelief, embarrassment, tearful and being visibly shaken. 
Citation: Lall S (2018) The Reality of Making a Medication Administration Error in Nursing Practice: Nurses Share Their Lived Experiences. Int J Nurs Clin Pract 5: 286. doi: https://doi.org/10.15344/2394-4978/2018/286

"...It was embarrassing and very painful as a human being...I was upset (for the patient) and I was embarrassed (for myself)."

"...I used to cry for everything...it was just like, 'Oh! My God!! This is the worst thing that could've happened to me...!

“...I was so scared! I was so scared!!! Because it's a blood pressure medication...

Nurses described the experiences to be emotionally draining and physically challenging as they threatened their personal and professional self. This, ultimately culminated into first a fear for the wellbeing of their patient and next a fear of losing their job. Nurses expressed concerns about the lack of collegiality and the environment of animosity that prevailed in workplaces which undermined their self-confidence to the extent that impeded their professional growth.

\section{Theme Category 2- Multiple Causes within Chaos: Cognitive Dimensions}

Nurses described multiple factors that impacted cognition which resulted in medication error occurrences. They spoke about practice challenges of being a new nurse, working in nursing homes with forty patients to one $\mathrm{RN}$, balancing multiple roles and responsibilities, workplace demands while caring for very sick patients. Nurses were concerned about the lack of support and an atmosphere of unfriendliness specifically experienced as newly hired nurses which made them unsure of themselves because they were expected to perform as experts even though they were novices with lack of support from their senior counterparts. Nurses also verbalized being physically and mentally exhausted with practically no breaks or recess due to the constant busyness and excess work demands.

"...being new to the system...considering the ratio of the patient...I had to give medication to forty patients... at 10 o'clock and there are a lot of them (medications)!"

“... as a new nurse there was time management, bullying, that is just there and then...just trying to learn your trade as well as the real-world side of it... and being so unsure of myself...it could happen to anybody..."

"...and if you have things on your mind, you are sleep deprived, you are tired, you are hungry you're (nurse) prone to make error..."

\section{ThemeCategory 3-EmbeddedChallenges:HealthcareSetting}

Nurses expressed concerns about the current healthcare system while describing experiences of constant struggle to meet multiple patient care demands in terms of patient care related situations which stemmed from disparities in work routines which were mostly related to a culture of unsupport prevalent among the leaders and peers and embedded in the work environment.

"...they (peers) start to pick at you and try to find things...it could really be an experience of true learning, but it's not... you are a nervous wreck ... they (peers) are like questioning this and that and trying to get you in trouble..."

\section{Theme Category 4- Organizational Culture: Within the Place, Within the Person}

Nurses described their experiences of an organizational climate that was mostly unaccepting and unsupportive of newcomers and was commonly rooted within the workplace and reflective of the behaviors of the nursing colleagues and leaders. Participants described experiences where they were afraid to approach senior colleagues for assistance or to voice concerns regarding patient care due to lack of camaraderie, support and collegiality.

"I remember being extremely tired, ... you are new, they're (co-workers) looking for any reason to get you in trouble... Well it was definitely always lack of support... they didn't want me there to begin with... a new grad, out of, out of... school! so of course they start to pick at you and try to find things...

“... a harsh scolding at the front desk, early in the morning...is highly inappropriate and should not have been done in that forum... this nurse already feels terrible, she is supposed to go home and this is what she goes home from..."

"It's all very, very stressful for a nurse! ...because she gives the medications, she or he, so it's ultimately that nurse's FAULT if there is an error made you know, it's very unfortunate!... they just have to put better practices in place...better staffing where they are supportive staff, not staff that are 'bullies'... They (nurses) don't need to be in an environment and be ridiculed!"

\section{Theme Category 5-Dynamics of Reflection: Looking Forward}

Nurses reflected upon experiences and identified multiple issues linked to the ever-changing dynamics of the healthcare system and the negative outcomes in terms of patient care and safety and its unfavorable impact on nurses professionally. Nurses described experiences that were linked to the contemporary organizational structure embedded in the healthcare facilities and expressed feelings of being treated unjustly by the leaders most of the times and not being

\begin{tabular}{|l|l|l|}
\hline Category of error/description & Participant \#s & Total occurrences in error category \\
\hline $\begin{array}{l}\text { Unauthorized drug error } \\
{ }^{*} \text { Drug given that was not ordered/needed to be held. }\end{array}$ & $1,3,4,5,6 \& 7$ & 6 \\
\hline $\begin{array}{l}\text { Wrong route error } \\
{ }^{*} \text { Drug administered to wrong area of body. }\end{array}$ & 1 & 1 \\
\hline $\begin{array}{l}\text { Wrong dose error } \\
{ }^{*} \text { Correct medication, incorrect dose. }\end{array}$ & 8 & 1 \\
\hline $\begin{array}{l}\text { Omission error } \\
{ }^{*} \text { Medication ordered was missed. }\end{array}$ & 3 & 1 \\
\hline $\begin{array}{l}\text { Wrong documentation error (New) } \\
{ }^{*} \text { Medication documented on patient who did not receive it. }\end{array}$ & 2 & 1 \\
\hline Table 2: Categories of Medication Errors. & & \\
\hline
\end{tabular}


heard in terms of factors that led to the medication error incidents. Nurses strongly expressed concerns about being held responsible for medication errors without being able to voice concerns and felt that such behaviors of organizational leaders will not serve to resolve issues and errors will constantly occur and nurses will continue to leave. Nurses also expressed strong concerns about unreceptiveness of organizational leaders to suggestions of relevant changes to the current healthcare system for improvement of future practice. Finally, nurses identified ways of fostering positive changes and just culture in the workplace environment to improve systems in healthcare facilities.

"... a lot of people...don't stay when they see the overload of work and the demand on the floor especially on a medical-surgical unit..."

“...Patient load hasn't really gotten better ...I moved from that 10:1, I thought that was too much! Spent three years in med-surg... There is a lot to do, just a lot to do! It's very you know demanding kind of work..."

"...incident reporting is not necessarily a bad thing but it's usually a reactionary thing in terms of med errors...to make it a proactive measure... a way to improve patient care or just put a positive spin on it... it's called an 'incident report'! ...it could also be... 'caution report' or something...just detaching the behavior from a person... that's what makes it a positive thing".

\section{Discussion of Findings}

Following is the discussion presented based on the findings of this study. The findings obtained were compared to the previous studies on medication errors and then the findings from this study were highlighted. The new findings that emerged from this study, although bear some similarities to findings of previous studies are unique in the way they were expressed by the participants of this study.

\section{Fear and anxiety, self-degradation}

Nurses verbalized feelings of extreme emotional and physical nature which resulted from situations after the medication error was committed. Nurses feared for the patient's wellbeing, but were equally anxious and fearful about being reprimanded, losing their job or professional license. Nurses also expressed thoughts of selfdegradation and remorse with respect to medication error occurrences and blamed themselves for the incidents. These feelings were similar to those expressed by nurses in other related studies $[2,3,18]$.

\section{"I was speechless...I really didn't know what to do!"}

\section{"I feel like I failed my patient that day..."}

Nurses, however, also spoke about multiple factors that contributed to these medication errors, particularly, the chaotic demands of the workplace with little or no organizational support. They said that if certain common issues were anticipated and addressed, the medication error/s could have been prevented.

Being overwhelmed and coping, being a new nurse and facing practice challenges

This study illuminated nurses' experiences of situations highlighting the complexity of workplace which was exceptionally distressing and overwhelming for nurses as they cared for patients while simultaneously trying to cope with difficult situations. Nurses expressed feelings of frustration and inadequacy when they were unable to complete patient related tasks and were constantly struggling to balance multiple $\mathrm{RN}$ responsibilities of patient care along with administering medications.

...when I was in med-surg for three years; it was so stressful...so overwhelming! ... you're dealing with different diagnoses, different treatment plans ...antibiotics every 6 hours... you have to draw blood, monitor vital signs... monitor a seizure ... patient with infected peg...changing dressings...it's just heavy task!”

Participants also stated that such experiences were mostly challenging when they were novices striving to familiarize themselves with the intricacies of the profession and simultaneously caring for patients of diverse nature. Nurses spoke about encountering stressful situations with colleagues and nurse leaders and experienced mistreatment and lack of guidance. Previous studies identified similar situations and experiences of nurses that reported inefficiencies, distractions, multiple factors and latent failures significant to error incidents [19-21].

Nurses spoke about altering their own behavior while transitioning from being new to becoming experienced by educating self about routines and policies while managing patient workload with little or no peer or leadership support and explained that these experiences were 'eye openers' both personally and professionally in terms of future practice. They described a culture that did not support the new nurses instead, the culture existing within the professionals was to primarily allow the new nurses to succeed or fail on an individual basis and not to offer individual support, acceptance or collaboration. The participants termed it as 'individual cultural sense', different but just as destructive as the culture of blame that was generally prevalent in the workplace. Nurses strongly advocated for support systems to be incorporated in workplaces where newcomers should be welcomed and encouraged by senior members of the nursing staff.

\section{Workplace culture, survival and moving on}

Nurses described a common atmosphere of censure and reproach encountered day to day during patient care both in the nursing homes and acute care facilities which was not just limited to medication error incidents. Some nurses described situations where they experienced behaviors of incivility by nurse managers and senior nurses towards them. Nurses emphasized that survival of new nurses in such a challenging work atmosphere is almost impossible and frequently forces new nurses to leave their jobs.

"... as a new nurse there was time management, bullying, that is just there and then, umm...just trying to learn your trade as well as the real-world side of it... and being so unsure of myself...it could happen to anybody...I've seen it happen to, you know, experienced nurses as well..."

Nurses were also, greatly concerned that because interruptions and distractions have become so commonplace and such a part of most workplaces in the healthcare arena, that leaders often fail to recognize these as significant factors that may result in most medication errors. Nurses in this study described experiences where they encountered and overcame challenging situations while caring for patients as they moved forward in the practice arena. Nurses also said that they 
understood the challenges of practice and recognized various barriers present in the current healthcare system and asserted that staff nurses must be included in discussions while addressing common patient issues for safe professional practice. Similar concerns by nurses were reported in previous studies addressing medication errors [22, $23,11,24,21]$

Nurses strongly expressed that patient care should not be an individual responsibility rather a team effort where members of the healthcare team may be able to depend on each other to deal with critical patient care situations owing to the busy and sensitive workplace environment and explicitly stated the need for change from a toxic environment to one that was just and supportive. Nurses attributed error occurrences to lack of collaboration among healthcare team members in terms of following correct protocol or individual team members following a different protocol or being unaware of the protocol. Nurses in this study stated that most organizational systems currently in place are possibly not very conducive to safe patient care practices, and strongly suggested the utilization of experienced frontline staff nurses in care related discussions to build future safe practices.

\section{Communication and teamwork, voicing concerns without fear}

Nurses in this study expressed concerns about not being able to voice concerns or express their opinions regarding medication errors or other patient care related issues because they were not invited to be a part of discussions vital to their patients.

"...definitely, get their (nurses) input, because they are the ones doing the medication administration, so they have to really be involved in the whole process ... input as far as the policies and procedures and umm...the nurses know the workflow, so you have to go to the nurses!... You have to talk to the nurses because they know what they actually did!”

This markedly draws attention to the fact that nursing is not involved directly in discourses or discussions where patient care concerns are addressed. This aspect is clearly reported by previous medication error studies $[3,25]$.

Participants in this study asserted that nurses' involvement in patient care and care related decisions should always be a primary focus which is less likely to be successful without effective communication and teamwork. Nurses stated that workplaces that lack communication and teamwork create major barriers for safe and effective patient care which invariably results in medication and other types of errors that compromise patient care. Nurses strongly believed that even though much system improvements have been made, medication errors are still prevalent because nurses are excluded from discussions related to these occurrences. Nurses uniquely and explicitly advocated that having conversations with those nurses who were involved in medication error incidents would be an excellent way to better understand the circumstances that predisposed to the medication error occurrences. Participants felt that nurses' input is most essential to equip units to reduce such incidents which, going forward would assist organizers and leaders to effectively improve existing work conditions as well as assist nurses to gain back selfconfidence and be able to voice concerns without intimidation from colleagues or supervisors.

\section{Governance and collegiality}

Nurses asserted that staffing decisions are commonly made by nurse leaders where nurses are frequently assigned to different set of patients on the same unit or moved out of the unit to staff another floor which disrupts the continuity of care and work routine. They (staff nurses), on the other hand are not allowed to organize or manage the care of their own patients. This disrupts teamwork and frequently leads to inconsistencies in work routine generating distractions that may promote error occurrences due to unfamiliarity of patients and patient care routines.

“...poor judgment and assigning too many patients with similar names to the same team... you have two nurses a team with 28 or 29 patients, we don't have a cap in the emergency room."

“... 'cause when you have a different nurse, she doesn't know the patient- she doesn't know the floor, she doesn't know the unit or the procedures, what goes on, on that floor, so she tends to be more fidgety and nervous and anxious!”

Literature reviewed reports several studies that have identified disruptions, distractions and interruptions as causes of medication errors and identifies them as the leading cause of medication error. Some studies recommend restructuring nursing and educating staff nurses as well as incorporating specific protocols to reduce medication errors [25-28].

This study highlighted participants' experiences where nurses explained that because patient care units were constantly busy it was extremely challenging to administer medications safely and simultaneously care for patients especially those that were critically ill with multiple care needs. Nurses expressed distress at the unfair treatment by peers while a few expressed gratitude for being treated fairly and said that support is much needed in times of crisis. Nurses strongly felt that difficult situations warranted coordinated teamwork and collegiality which would help significantly to manage patient care and avoid medication error incidents. Participants suggested involvement of nurses in creating processes for safe medication administration and said that nurses are the persons who understand the medication administration process better than anyone else. Nurses also stated that because they are often the second victims who experience the errors along with patients, they should be the designated change agents to help promote safe medication administration process. Nurses further asserted that adequate staffing as well as a collegial and supportive workplace environment free of intimidation is extremely essential to reduce medication errors.

\section{Professional security}

This study revealed nurses' concern about the wellbeing of their patients when a medication error/s occurred because they were aware of their responsibility towards the safety of patients. However, nurses were also extremely concerned about their professional security which was mostly generated from a fear of being held accountable if patient was harmed by the error and the culture of blame prevalent in the workplace.

“...people are afraid of losing their jobs, so they're not gonna say anything or they'll just be prone to making more errors because the environment is not conducive to one's learning, for safe practice (blame) and for people to feel comfortable...to say, "ok I made a booboo, don't, don't kill me!" 
All participants in this study agreed that underreporting of medication errors was largely due to an underlying fear of losing one's job. These findings were identified by previous medication error studies $[20,29,2]$.

Most participants in this study described a general atmosphere of hostility in the workplace where nurses were against nurses and the workplace culture was that of animosity and antagonism towards each other. Nurses felt that one wrong move may jeopardize their job because they were either new to practice or new to the unit and were constantly made to feel that they needed to prove themselves to their colleagues. Nurses felt that they were being set up for failure which rendered them incapable of handling patient situations professionally. Nurses described feelings of being unwanted and undeserving of the $\mathrm{RN}$ position mostly after the medication error incident and felt that senior nurses tried to make them look incompetent in the sight of the nurse manager as if they committed a misdemeanor. Nurse colleagues often tried to implicate them in multiple ways which generated insecurity and a fear of losing their professional status. Participants believed that maintaining a job status was more important than being hired as a RN for the first time. Nurses in this study strongly suggested that healthcare leaders need to strengthen frontline staff nurses by eliminating the culture that exudes constant criticism, reprimand and rebuke and inculcate the workplace environment with collegiality and support.

\section{Study Limitations}

This study was conducted in the USA, state of New York therefore; the transferability of the results globally may be questioned due to variations in the healthcare systems prevailing in other countries. Another possible limitation of this study may be related to the selfselected sample, which may be viewed as subject to bias as the nurses involved in medication errors may have projected exclusive individual attitude owing to the unique nature of these experiences. Additionally, those nurses who volunteered to participate in this study might have demonstrated a strong interest in medication safety. Meanwhile, those nurses who were less confident and forthcoming in wanting to share their experiences may have been unintentionally excluded from this study. Finally, the researcher's professional experience as a nurse of several years with multiple roles and responsibilities may be viewed as subject to bias.

\section{Implications and Recommendations}

\section{Nursing practice and healthcare organizations}

It is common knowledge that making errors is part of the human experience therefore; nurses and other healthcare professionals being humans may commit medication errors. According to Reason [30] there are two ways to view causes of human errors. The person approach attributes the errors to individuals (culture of blame) and the system approach assess deficiencies in the existing systems and recognize individuals to be fallible (just culture). Research suggests that perceptions of a safe patient care environment and safety culture in healthcare facilities vary among staff nurses and nurse leaders because of differences in their viewpoints which is a major deterrent to quality patient care and a concern among staff nurses [31,32]. Other medication error studies were found to be linked to lateral violence and bullying $[33,34]$.

Present-day healthcare system has metamorphosed in numerous ways, the most significant of which is the eminence of RN roles and responsibilities of patient care. This transformation calls for frontline nurses to be leaders at the bedside with full dominion and responsibility of patient care. The findings of this study suggest that nursing practice and healthcare organizations consider the following recommendations:

- Provide at all times, adequate staffing on units, not only based on numbers but also consider the acuity and diversity of the patient population to enable prioritization of care and teamwork while providing safe and quality care to all patients.

- Remodel workplace culture to champion collegiality among all members of the nursing team as well as among the interdisciplinary healthcare team members for better and improved patient outcomes.

- Restructure to build a non-toxic, blame free and stress-free culture in workplaces enabling nurses to voice patient related concerns and be change agents for better patient outcomes.

- Organizational and nurse leaders may create and/or incorporate programs that provide staff nurses with learning experiences of high caliber and quality for the continued enhancement their management skills to encourage professional growth and safe quality patient care.

\section{Nursing education}

Being that nursing students have restricted and supervised exposure to patients, it is a common observation that they acquire very limited experience in the care of patients. Therefore, it is vital that new graduate nurses should be guided and supervised by experienced nurses, especially in the first year of nursing practice $[35,36]$. Participants in this study revealed new realizations with implications for nursing education that underscored the importance of educating student nurses who are about to graduate in a way that they can anticipate and overcome obstacles while entering practice to reduce medication error incidents. The findings of this study recommend and/or suggest that nursing educators may consider the following recommendations:

- Qualitative medication error studies about nurses' lived experiences may be conducted in other states of the USA, other countries and globally for a varied perspective of medication errors in other healthcare systems currently across the USA and worldwide.

- Quantitative medication error studies to compare and correlate medication errors to staff nurse concerns about patients and nurses across the healthcare systems.

- Research studies to further investigate medication error categories to understand what really constitutes a medication error in the administration process and staff nurse involvement.

- Studies for in-depth investigation of medication errors from the perspective of student concerns to understand challenges of transition into practice. This may assist nurse educators to formulate ways for seamless transition of new nurses into practice.

\section{Nursing Research}

Research suggests that there is paucity of studies related to nurses' lived experiences and perceptions of making medication errors in the USA and globally. The following are recommendations to consider for further research: 
Citation: Lall S (2018) The Reality of Making a Medication Administration Error in Nursing Practice: Nurses Share Their Lived Experiences. Int J Nurs Clin Pract 5: 286. doi: https://doi.org/10.15344/2394-4978/2018/286

- Qualitative medication error studies about nurses' lived experiences may be conducted in other states of the USA, other countries and globally for a varied perspective of medication errors in other healthcare systems currently across the USA and worldwide.

- Quantitative medication error studies to compare and correlate medication errors to staff nurse concerns about patients and nurses across the healthcare systems.

- Research studies to further investigate medication error categories to understand what really constitutes a medication error in the administration process and staff nurse involvement.

- Studies for in-depth investigation of medication errors from the perspective of student concerns to understand challenges of transition into practice. This may assist nurse educators to formulate ways for seamless transition of new nurses into practice.

\section{Conclusion}

Research suggests that even though healthcare organizations continue to diligently work towards improving the existing systems, medication errors continue to be the highest and most common predictable cause of medical errors of which administration errors are the leading cause of all medication error occurrences. This poses a significant question for the healthcare organizations and leaders to burrow beyond the surface to identify and address the factual causes of medication errors that are embedded within individual organizational setups and develop novel ways to resolve these issues. This study identifies important issues that are relevant to medication errors and recommends ways to reduce medication error incidents through the descriptions of the lived experiences of nurses that were involved in medication errors to build a future of a safer patient care environment in contemporary healthcare systems.

\section{Competing Interests}

The author declares that no competing interest exists.

\section{References}

1. Husserl E (1965) Phenomenology and the crisis of philosophy. New York, NY: Harper \& Row.

2. Schelbred A, Nord R (2007) Nurses' experiences of drug administration errors. J Adv Nurs 60: 317-326.

3. Treiber LA, Jones JH (2010) Devastatingly human: An analysis of registered nurses' medication error accounts. Qualitative Health Research, 20: $1327-$ 1342.

4. Institute of Medicine (2000) To err is human: Building a safer healthcare system. National Academy Press, Washington, DC

5. Institute of Medicine (2006) Preventing medication errors. Washington DC National Academy Press.

6. Institute of Medicine of the National Academies (2007) Preventing medication errors. Washington DC: The National Academies Press.

7. National Medicines Information Centre (2001) NMIC bulletin on medication errors 7: (1-4).

8. Cousins D, Dewsbury C, Mathew L, Nesbitt I, Warner B, et al. (2007) Safety in doses: Medication safety incidents in the NHS. National Patients Safety Agency, London.

9. Joolaee S, Hajibabaee F, Peyrovi H, Haghani H, Bahrani N, et al. (2011) The relationship between incidence and report of medication errors and working conditions. Clinical Issues 58: 37-44.

10. Bates DW, Spell N, Cullen DJ, Burdick E, Laird N, et al. (1997) The costs of adverse drug events in hospitalized patients. Journal of the American Medical Association 277: 307-311.
11. Buchini S, Quattrin R (2011) Avoidable interruptions during drug administration in an intensive rehabilitation ward: Improvement project. $J$ Nurs Manag.

12. Pepper G (1995) Errors in drug administration by nurses. Am J Health Syst Pharm 52: 390-395.

13. Classen DC, Pestotnik SL, Evans RS, Lloyd JF, Burke JP, et al. (1997) Adverse drug events in hospitalized patients: Excess length of stay, extra costs, and attributable mortality. JAMA 277: 301-306.

14. Streubert HJ, Carpenter DR (2011) Qualitative research in nursing: Advancing the humanistic model (5th ed.). Philadelphia: Wolters Kluwer/ Lippincott Williams \& Wilkins.

15. Colaizzi PF (1978) Psychological research as the phenomenologist views it. In R. Valle \& M. King (Eds.). New York, NY: Oxford University Press.

16. Shenton AK (2004) Strategies of evaluating trustworthiness in qualitative research projects. Education for Information 22: 63-75.

17. Lincoln YS, Guba EG (1985) Naturalistic inquiry. Newbury Park, CA: Sage Publications.

18. Kim KS, Kwon SH, Kim JA, Cho S (2011) Nurses' perceptions of medication errors and their contributing factors in South Korea. J Nurs Manag 19: 346353.

19. Elganzouri E, Standish C, Androwich I (2009) The mat study: Global insight into medication administration process. Stud Health Technol Inform 146: 424-428.

20. Osborne J, Blais K, Hayes JS (1999) Nurses' perceptions: When is it a medication error? J Nurs Adm 29: 33-38.

21. Lawton R, Carruthers S, Gardner P, Wright J, McEachan RR, et al. (2012) Identifying the latent failures underpinning medication administration errors: An exploratory study. Health Serv Res 47: 1437-1459.

22. Biron AD, Lavoie-Tremblay M, Loiselle CG (2009) Characteristics of work interruptions during medication administration. J Nurs Scholarsh 14: 330336.

23. Agyemang R, While A (2010) Medication errors: Types causes and impact on nursing practice. Br J Nurs 19: 380-385.

24. Dickson GL, Flynn L (2011) Nurses' clinical reasoning: Processes and practices of medication safety. Qual Health Res 22: 3-16.

25. Gibson $T$ (2001) Nurses and medication error: A discursive reading of the literature. Nurs Inq 8: 108-117.

26. Pape TM (2003) Applying airline safety practices to medication administration. Medsurg Nurs 12: 77-93.

27. Petrova E (2010) Nurses' perceptions of medication errors in Malta. Nursing Standard 24: 41-48

28. Hayes C, Jackson D, Davidson P, Power T (2015) Medication errors in hospitals: a literature review of disruptions to nursing practice during medication administration. J Clin Nurs 24: 3063-3076.

29. Antonow JA, Smith AB, Silver MP (2000) Medication error reporting: A survey of nursing staff. J Nurs Care Qual 15: 42-48.

30. Reason J (1995) Understanding adverse events: Human factors. Qual Health Care 4: 80-89.

31. Vogelsmeier A, Scott-Cawiezell J, Miller B, Griffith S (2010) Influencing Leadership Perceptions of Patient safety Through Just Culture Training. J Nurs Care Qual 25: 288-294.

32. Stevens KR, Engh EP, Tubbs-Cooley H, Conley DM, Cupit T, et al. (2017) Operational failures detected by frontline acute care nurses. Res Nurs Health 40: 197-205

33. Roche M, Diers D, Duffield C, Catling-Paull C (2010) Violence toward nurses, the work environment, and patient outcomes. J Nurs Scholarsh 42: 13-22.

34. Vessey JA, DeMarco R, DiFazio R (2011) Bullying, Harassment, and Horizontal Violence in the Nursing Workforce. Annu Rev Nurs Res 6: 133158.

35. Benner P (1984) From novice to expert: excellence and power in clinica nursing practice. Menlo Park, CA: Addison- Wesley.

36. Benner P, Tanner C, Chesla C (1992) From beginner to expert: Gaining a differentiated clinical world in critical care nursing. ANS Adv Nurs Sci 14: 13-28. 